\title{
The Era of Covid-19; Its' Impact on Cancer Patient Experience in Southwest Nigeria
}

\author{
Aisagbonhi Henry Osaro ${ }^{1 *}$, Daniel Olusoji $\mathrm{J}^{2}$, Esther Ajakaiye $\mathrm{A}^{3}$, Ogunyemi A.A ${ }^{1}$, \\ Adaraloye J.O ${ }^{1}$, Odewabi A.O. ${ }^{1}$, Adenuga J.O ${ }^{1}$, Adegbuyi S.O. ${ }^{1}$, Adetogun T.A ${ }^{1}$, Ofomola \\ Harlims $^{4}$, Orisade Joel ${ }^{1}$ \\ ${ }^{1}$ Laboratory Services Department, Olabisi Onobanjo University Teaching Hospital, Ogun \\ State, Nigeria \\ ${ }^{2}$ Olabisi Onobanjo University Teaching Hospital, Sagamu. Community Medicine and \\ Primary Care, Nigeria \\ ${ }^{3}$ Physiology Department, University of Ibadan, Oyo State, Nigeria \\ ${ }^{4}$ Laboratory Services Department, Delta State University, Teaching Hospital Oghara, Delta \\ State, Nigeria
}

\begin{abstract}
Patients have developed a well-informed sense of validation concerning maintaining hygiene standards. The pandemic, somehow in disguise, presented the valuable perspective of life reflection and gratitude. The attitude of a majority of patients engaged was immensely that of a deep reflection of the privilege of having access to early diagnosis, treatment, and adequate support. However, the complaints of the past, knowing that they are alive and have hope to keep fighting on with gratitude and sense of acceptance. However, they wished that the experience of the social media - health engagement platform should continue as it provided a good level of the bridge of gap of information. The cost of treatment remains a major concern as it largely translates to possible dropout from treatment courses for most cancer patients who can't afford the current cost. The concerns of the cancer patients and survivors during and after the covid-19 pandemic are similarly a concern to public health professionals worldwide. The need to aid their return to routine health care services is more important and therefore requires an urgent reorganization of cancer management services. An urgent intervention should be focused on patient re-orientation /pandemic control, staff training and retraining, awareness campaign, screening and result accessibility, special pandemic services, amongst others. The outcome reflected a very huge level of poor cancer patient experience in the public cancer treatment centres, while the reverse is the case with the private cancer treatment centres. This can be managed if an urgent intervention as proffered is implemented.
\end{abstract}

Keywords: Cancer, Coronavirus, Covid-19, Cancer screening, Cancer treatment, Patient experience.

\section{Introduction}

Coronavirus are important human and animal pathogen. In 2019, a novel coronavirus was identified using open isolation and sequencing. The virus is called the severe acute respiratory syndrome coronavirus 2 (SARSCoV-2), which causes COVID-19. It is known to be highly contagious, and the current vaccine is not known to cause long-lasting immunity. Over 1 million people have died due to COVID-19. So far, the disparities in incidence as well as mortality rates make control difficult. This situation has presented completely a new normal to every sphere of life in terms of relationships and interactions [1]. The COVID19 pandemic has severely impacted all areas of life. The medical care institutions have been 
severely affected as well. Cancer patients have been affected in terms of the treatment delivery care with the upsurge of competing risk, the cancer patients are immune-compromised, and this puts them at a greater risk of infection or dying from it. There is also the problem of interruption of care arising from competing availability of manpower and the new COVID19 influenced treatment framework. There is also the case of the current alarming rate of lack of resources at the national and state level due to the reshuffling of resources to cater for distribution across the board.

At this juncture, there is no one size that fits all in terms of the current approach to delivering care to all the various cancer types.

Some occurred significant changes to the delivery of cancer care include.

1. Social distancing in clinic waiting rooms and work areas.

2. New and continuous experimenting screening procedures and laws.

3. Drive-through blood draw and injections which minimizes contacts.

4. Inability to offer or access treatment due to travel restrictions.

5. Engagement of telemedicine and virtual check-ins which leaves a greater proportion of cancer patients without access to information technology without access to periodical consultation.

The sudden pandemic of SARS-Cov-2 (also known as novel coronavirus disease 2019,
COVID-19) poses a severe threat to hundreds of millions of lives in the world [2]. They further affirmed that the complete cure of the virus largely relies on the immune system, which becomes particularly a challenge for the cancer subjects, whose immunity is generally compromised. However, there is a general consensus that the clinical data on the prevalence of SARS-Cov-2 for cancer patients is still limited [2]. Patients with Cancer remains one of the groups that are most affected by the COVID-19 pandemic. The reasons include that the Frequent systemic immunosuppressive state and the requirement of frequent admission to the hospital.

It is obvious that cancer diagnosis is very likely to increase during and after the COVID19 pandemic as a result of halted screening at virtually most health facilities across the country. In fact, the frequent visits to the hospital will also increase the rate of exposure at contacting the Covid-19 virus [3, 4]. In lieu of this, there is the need to think ahead at this time and unconsciously develop an evidencebased guideline for the management of cancer patients during and beyond the pandemic. In doing this, the patient experience will play an important, informed part of the evidence-based guideline. In recent conferences, the demanding questions now are if Cancer increases the risk of contracting COVID-19 or the other way around [5].

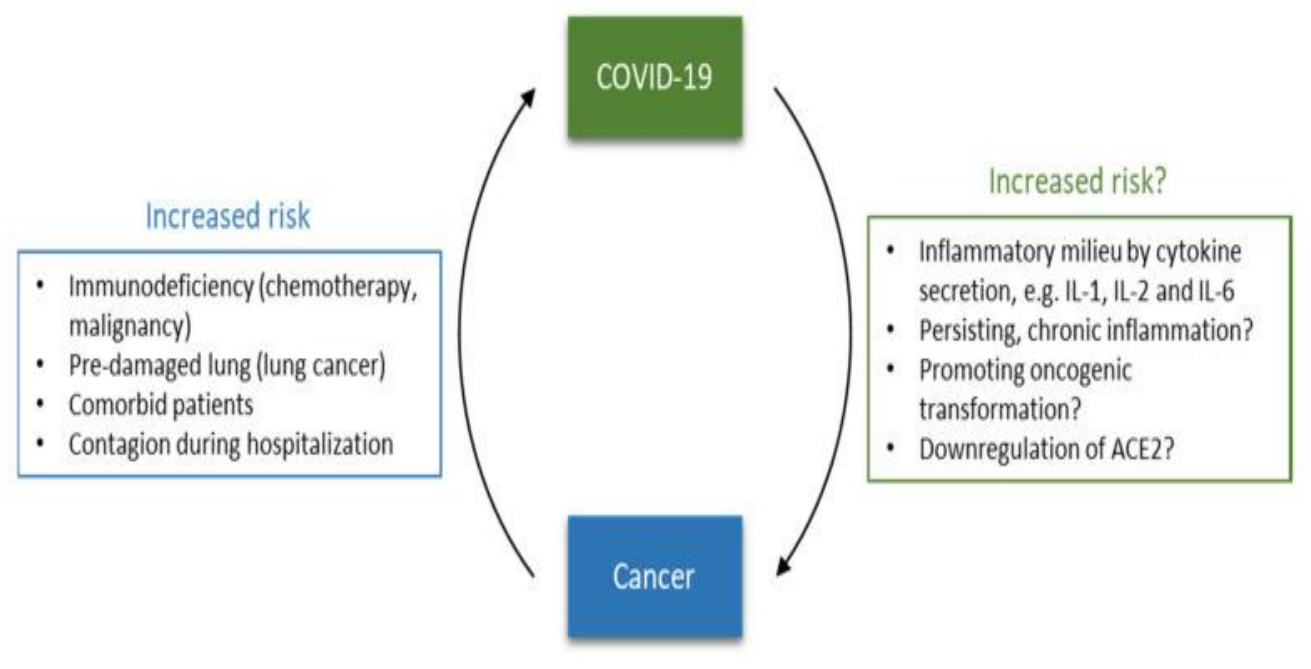


Figure 1. Bidirectional relationship between coronavirus disease 2019 (COVID-19) and cancer

\section{Increasing Risk: Covid-19 and Cancer}

Table 1. Oncoviruses and respective Cancers

\begin{tabular}{|l|l|}
\hline Virus & Cancer \\
\hline EBV & $\begin{array}{l}\text { Burkitt's lymphoma, non-Hodgkin lymphoma, post-transplant } \\
\text { lymphoproliferative disorder, nasopharyngeal carcinoma }\end{array}$ \\
\hline HPV & Cervical carcinoma, Head, and neck cancer \\
\hline HBV & Hepatocellular Carcinoma \\
\hline HCV & Hepatocellular Carcinoma \\
\hline HTLV1 & Adult T-cell leukemia \\
\hline KSHV & Kaposi's sarcoma \\
\hline MCPyV & Merkel cell carcinoma \\
\hline HCMV & Mucoepidermoid carcinoma \\
\hline
\end{tabular}

EBV (Epstein-Barr virus), HPV (human

of 2020, Cancer currently accounts for 8.8 papilloma virus), $\mathrm{HBV}$ (hepatitis virus $\mathrm{B}$ ), $\mathrm{HCV}$ million deaths annually. The data is that $70 \%$ of (hepatitis virus C), HTLV-1 (Human T-cell this mortality is from Africa and Lymphotropic Virus), KSHV (Kaposi's Sarcoma Herpesvirus), MCPyV (Merkel Cell Polyomavirus), HCMV (human cytomegalovirus).

Currently, researchers are actively seeking underdeveloped Nations / low and middleincome countries.

Incidence is 1 in 5 man and 1 in 6 women, while mortality is 1 in 8 men and 1 in 11 women (IARC, 2020). answers to the possible causal relationship. As

Estimated number of new cases in 2020 , worldwide, females, all ages

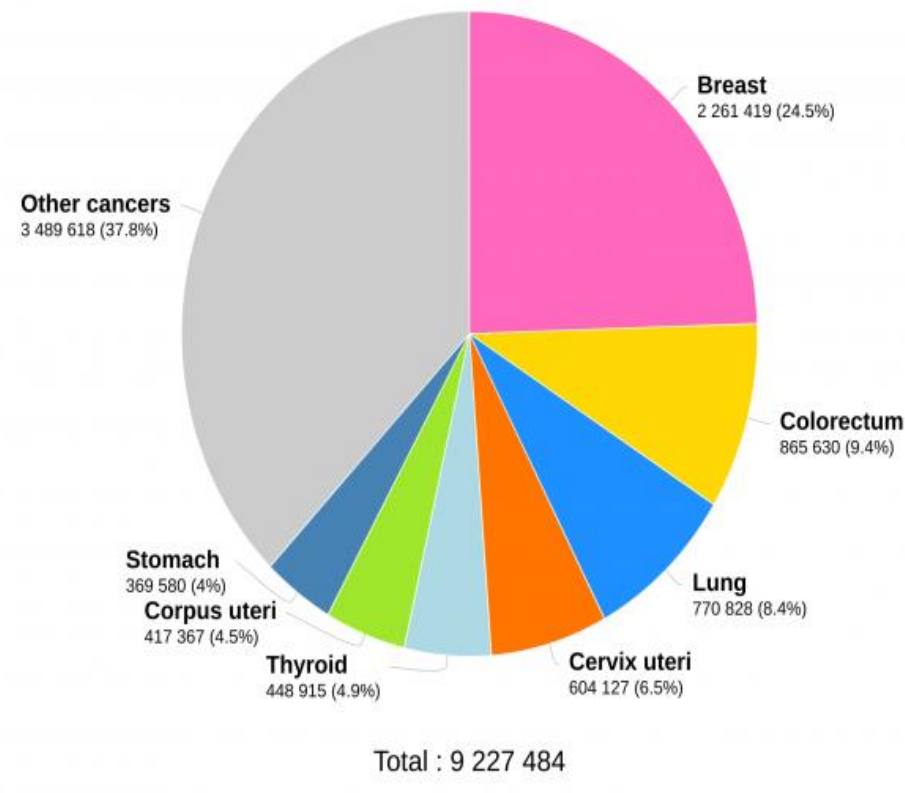

Figure 2. Estimated Number of New Cases in 2020. Worldwide, Females, all Ages 


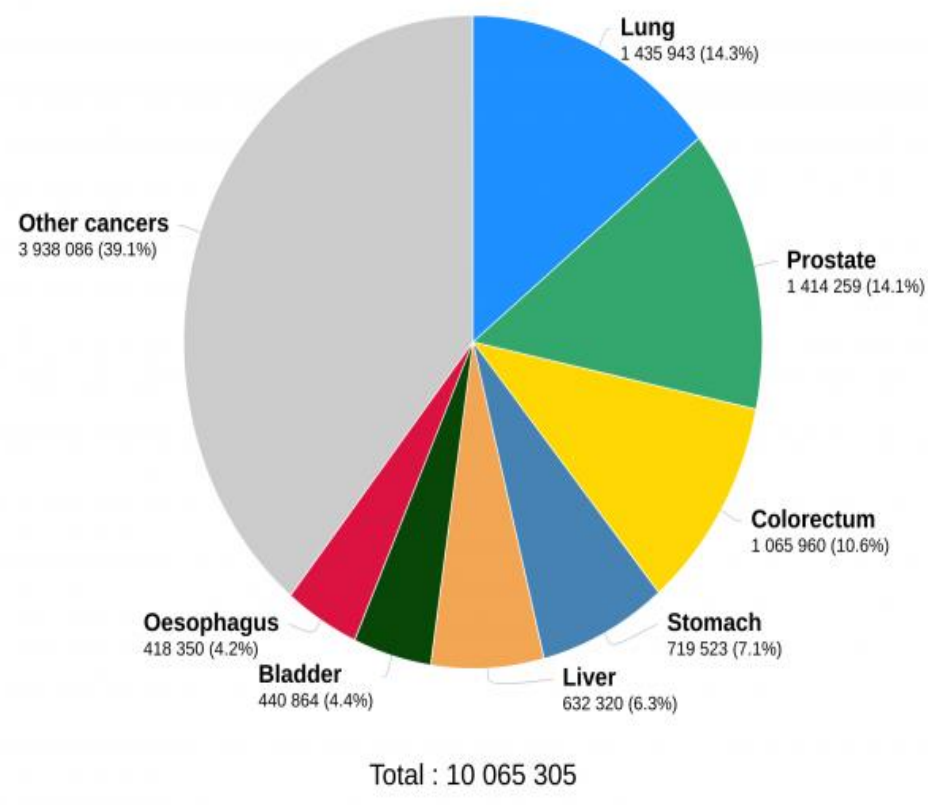

Figure 3. Estimated Number of New Cases in 2020. Worldwide, Males, all Ages

It is a global public health challenge in demand for a new delivery care approach in the epidemic era.

Asia and Africa have a higher proportion of cancer deaths compared with their incidence. This is due to the prevalent higher frequency of cancer types with poor prognosis and the unfortunate situation of limited diagnosis and treatment.

The demand for an improved cancer delivery care approach in Nigeria and Africa is also due to the possible certainty of increased diagnosed cancer cases which is consequent to the increasing population growth worldwide and ageing, which is a major factor.

The predisposing risk factor of Cancer is also on the rise and playing a big part. An example includes obesity, diabetes mellitus, respiratory disorders, etc.

Finally, in the UK (United Kingdom), the 2014 UK population stood at 65 million.

The 2035 estimated UK population is 73 million. However, in 2014, people diagnosed with cancer in the UK were 360,700 .

In Nigeria, the total number of cancer cases as of 2018 stood at 115,950 , while the deaths recorded were 70,327 . This is a huge impact compared to a more catastrophic impact with COVID-19 pandemic.

The prediction in 2035 is an additional 12,600 cancers due to risk factor changes 140,600 will be cancelled due to population changes leading to a total estimated case of 514,000 . 


\section{WHY ARE WE EXPECTING MORE PEOPLE TO BE DIAGNOSED WITH CANCER?}

It's mostly because the population is growing and getting older, but risk factors that cause cancer are also playing a bigger part.

UK POPULATION

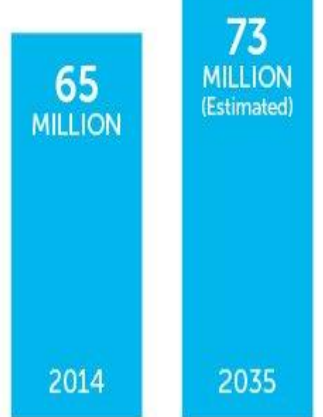

Source: cruk.org/cancerstats LET'S BEAT CANCER SOONER cruk.org

PEOPLE IN THE UK DIAGNOSED WITH CANCER

2014 in $\quad$ 巾 $=5,000$ prin in in

Total cases in 2014 360,700

\section{5 ำ III

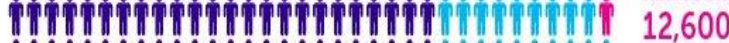

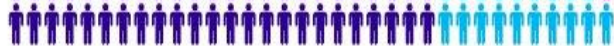

due to population changes

Estimated total 140,600

cases in 2035

514,000

CANCER

Figure 4. The 2035 Estimated UK Population Diagnosed with Cancer

Adapted from cancer research UK, cruk.org/cancerstats

NIGERIA

BURDEN OF CANCER

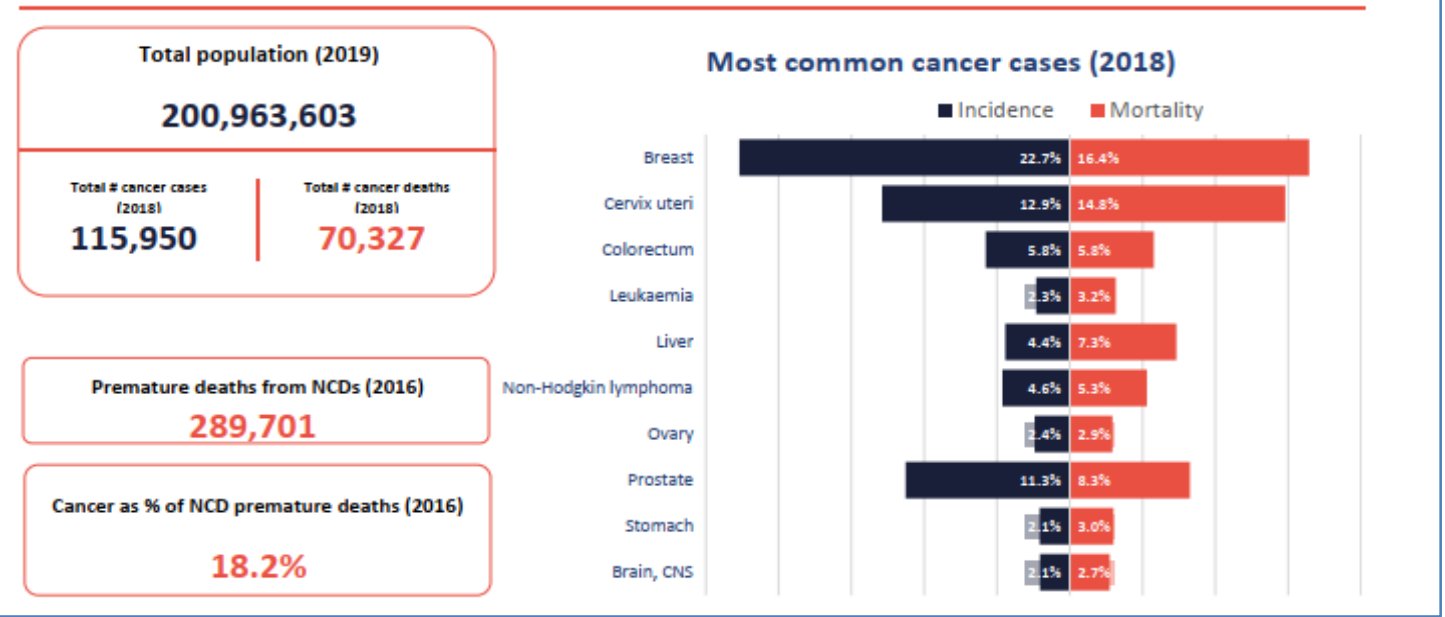

Figure 5. Cancer Country Profile 2020. Nigeria

Adapted from www.who.int.

In Africa, there is a high rate of lack or inadequate cancer research capacity due to lack of funds and under-funding. Apart from colorectal, breast, and prostate cancer, lung cancer alone accounts for $13 \%$ of all new cancer diagnosis globally and $24 \%$ of cancer deaths.

Currently, lung cancer research needs investment that matches the impact of the disease. 
In Nigeria, people living in the rural areas and with low socio-economic status are worse likely to not have access to diagnosis compared to persons living in the urban areas during the pandemic.

\section{Incidence and Cancer in Numbers during Pandemic}

The paucity of data in Nigeria regarding this field is a challenge. However, considering other data, the National population-based modelling study from England found a substantial increase in the number of available cancer deaths in England are to be expected; 7.9 to $10 \%$ breast cancer, 15.3 to $16 \%$ lung cancer, 4.8 to $5.3 \%$ for oesophageal Cancer.

This is due to delay in treatment waiting periods, inadequate access, and travel restrictions. This data is worrisome, and if to be applied to Africa, the similar ripple outcome is far obvious considering the level of our countries health status.

According to a JAMA study of more than 200,000 cancer patients looked at during the pandemic and the current incident rate of many types of cancers, there was a significant decline in newly identified patients with six common types of Cancer.

The findings were similar to studies from the Netherland cancer registry $(40 \%$ decline in weekly cancer incidence and the UK $(75 \%$ decline in referral for suspected COVID-19 restrictions were implemented) [6]. The decline in newly identified cancer patients in Nigeria was also obvious following the many months of restrictions and state lockdowns across the nation.

\section{$20 \%$ rise in cancer deaths expected in England due to Covid-19 emergency}

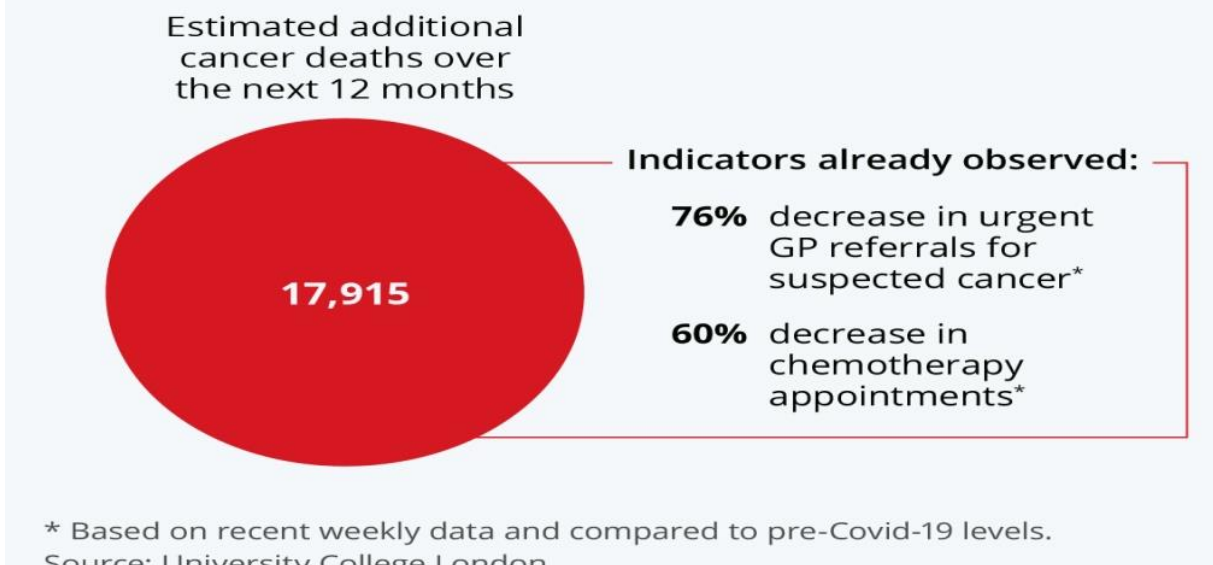

Source: University College London

\section{statista}

Figure 6. Expected Rise in Cancer Deaths

Adapted from https://www.statista.com/chart/21550/cancer-deaths-england-coronavirus/

\section{The CDC Guidelines on Providing Safe Care for Patients during Pandemic}

1. In general, any clinical visits that can be postponed without risk to the patient should be postponed; similar visits include routine surveillance biscuits visits to detect cancer reoccurrence (mammograms are colonoscopy).

2. Screening clinics should be developed to allow for patients with symptoms to be 
evaluated and tested in dedicated units with dedicated staff.

3. Patient screening status \& Covid19 positive status should be documented prior to the patient entering the facility.

Other CDC guidelines are on:

1. General health care facility and healthcare professional guardians.

2. General clinical care guidelines.

3. Home Care guidelines.

4. High risk for population guidelines.

Cancer Screening, Diagnosis, and Staging during Pandemic

The halt in national screening programs in most countries during the pandemic and its impact on future cancer mortality is a matter on the front burner. The backlog of patients with symptoms would happen and need urgent assessment therefore, assistance will have to be prepared for in the nearest future [2].

1. There are definitely the increasing delayed elective surgeries that need to be managed.

2. Elective surgeries as to when it should be resumed is dependent on,

a. Infection rates and downward rates patterns.

b. Availability of resources for utilization

c. Possible privatization and scheduling of cases managed by all stakeholders' example surgeons, general doctors, theatre nurses etc.

Practical Approach to the Management of Cancer Patients and Anticancer Treatments during Pandemic Using the Pandemic in 2019

On top of a wide range of medical references and interim guidelines, including $\mathrm{CDC}, \mathrm{NCI}$, ASCO, ESMO, NCCN, AACR, ESMO, and the National Health Commission of China, etc., they formed a guideline based on their experience in some specialized cancer hospitals (Tables-7, 8 and 9) in Wuhan- the originally endemic center of the virus. Furthermore, they formulated an expert consensus which was developed by all contributors from different disciplines after full discussions based on their understanding and analysis of limited information of COVID-19. The consensus highlighted a multidisciplinary team diagnostic model with the assessment of the balance between risks and benefits prior to treatment, individualizing satisfaction of patients' medical needs, and acceptability in ethics and patients' socio-economic conditions.

In Nigeria, evidence of similar policies at the national level don't seem to be accessible, and perhaps non-existence as detailed search and enquiry has yielded no outcome. However, the Nigeria National Policy on cancer control has been established, but to what extent is it been implemented to adjust to the present pandemic' is yet to be observed at the grass root.

\section{Model for Covid-19 Screening in Oncology}

1. Cancer + symptoms or exposure to positive persons $=$ priority testing.

2. Cancel + no symptoms $=$ variable across institutions.

3. All lung cancer patients should be tested.

4. Cancer patients with lower respiratory symptoms and/or signs (e.g., Fever, cough, dyspnea, or hypoxia.) All those with exposure to someone with concerned covid19, will proceed with testing for SARScov-2 using a nucleic acid amplification test.

5. Cancer patient with a known exposure need to be tested for covid-19, quarantined, and closely followed up. 


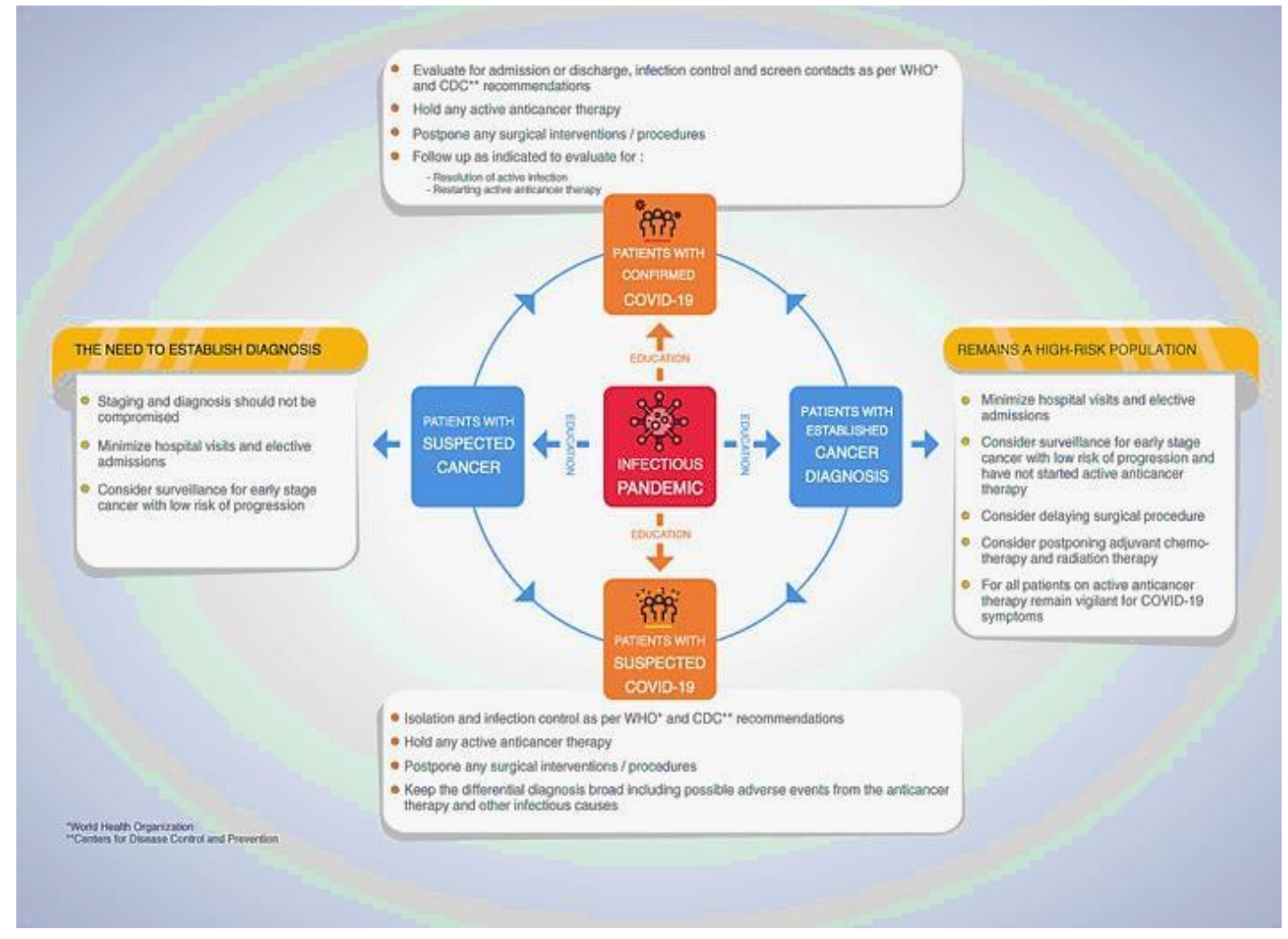

Figure 7. Practical Approach to the Management of Cancer Patients with Suspected COVID Symptoms in Covid-19 Era

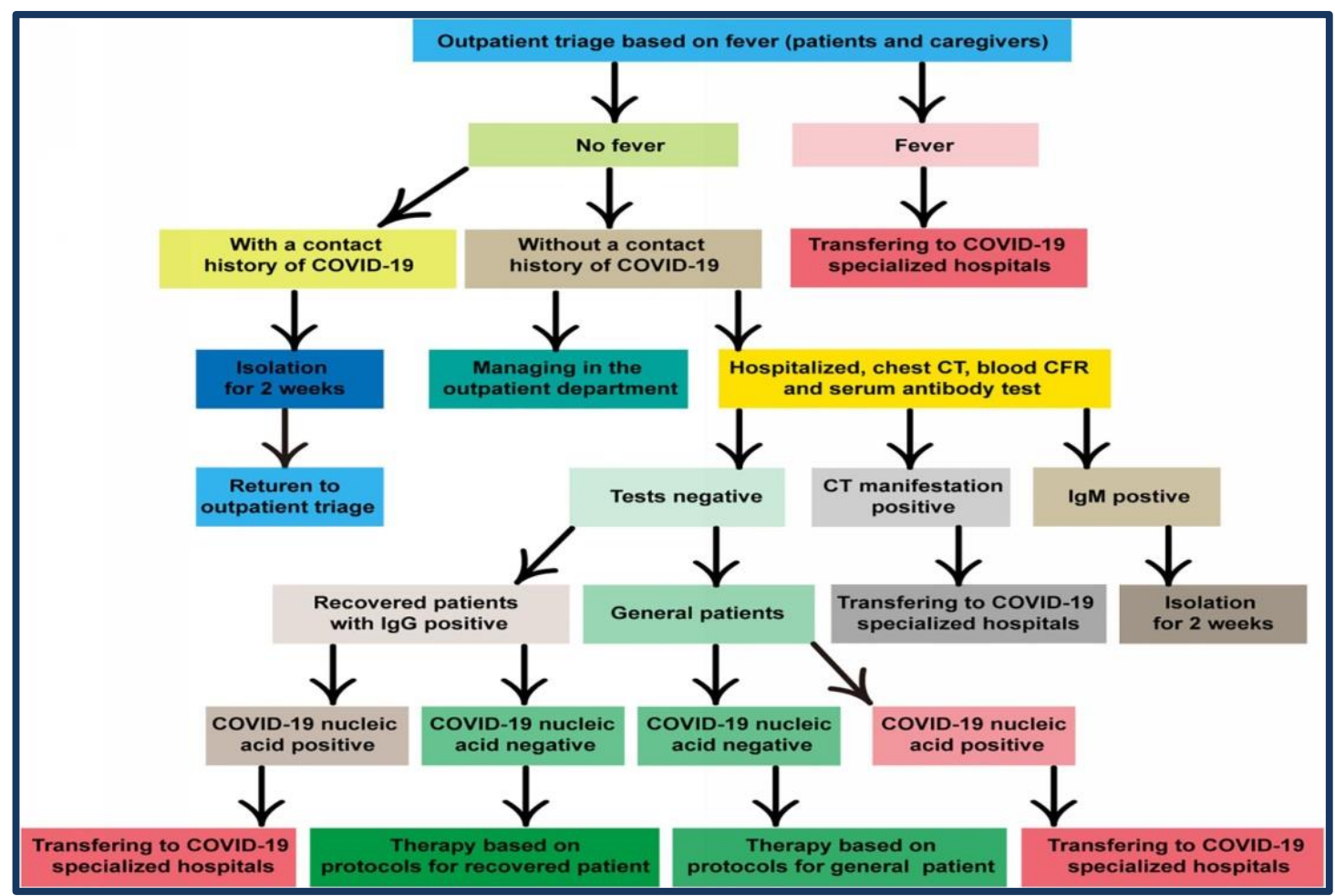

Figure 8. Practical Approach to the Management of Cancer Patients with Fever in Covid-19 Era 


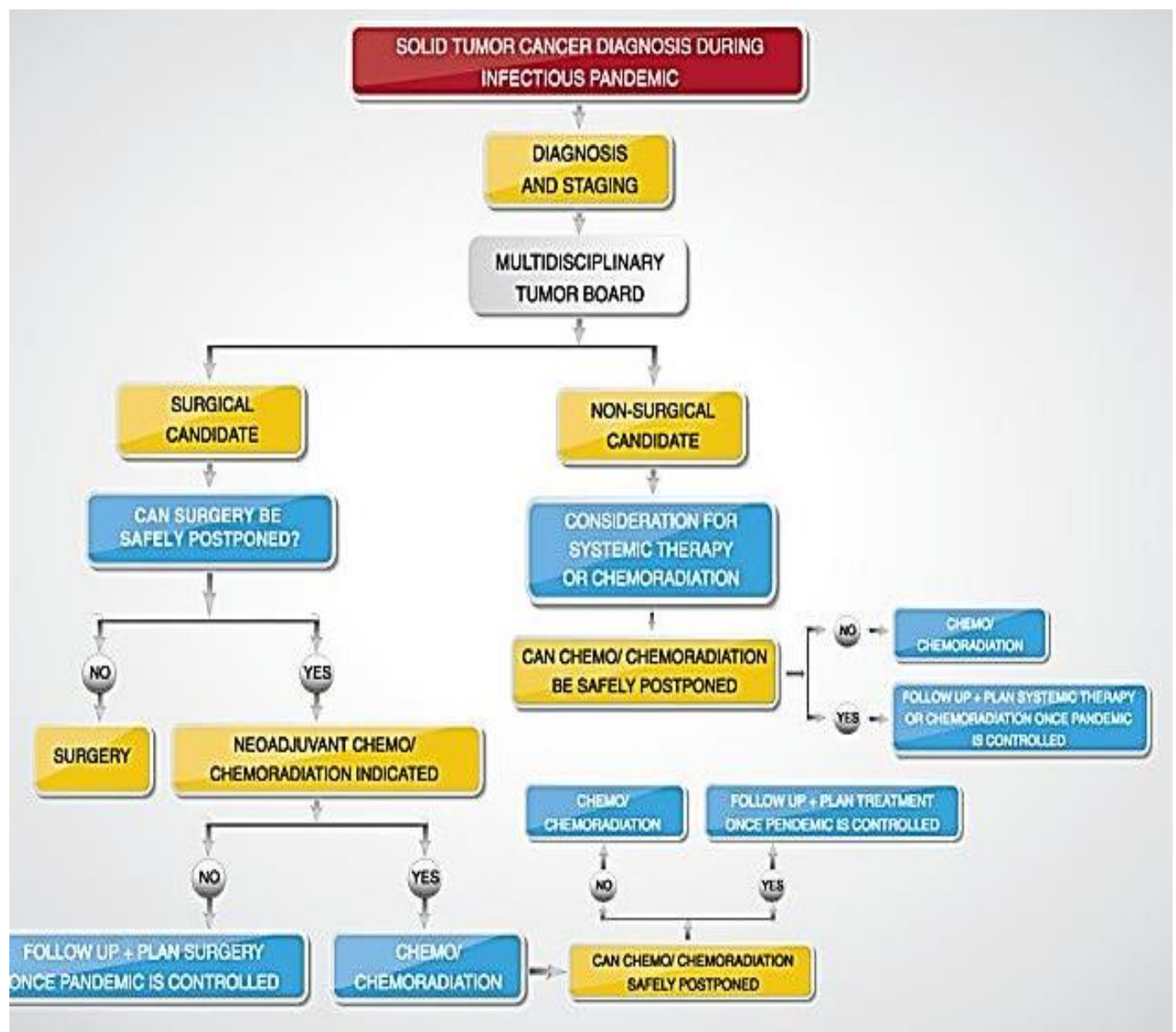

Figure 9. Practical Approach to the Management of Cancer Patients with Solid Tumour Diagnosis in Covid-19 Era

Adapted from the practical approach to the management of cancer patients during the novel coronavirus disease in 2019 pandemic: an international collaborative group [7].

\section{Dealing with Cancer Patients Mental Health during the Covid-19 Pandemic}

Mental health is a big aspect of cancer patients and cancer care. patient presents stress, depression, anxiety, insomnia, denial, anger, and fear on the other hand, studies have shown that more than $53 \%$ are worried $45 \%$ so quiet and understanding, $42 \%$ are anxious $33 \%$ as stressed and about $22 \%$ are depressed.

The mental health of caregivers is also very crucial. Physicians, nurses, laboratory scientists, and others seem to be borne out and appear isolated due to long straight work hours as they worry about their own health and meeting with the complex demands at work as well as difficult choices inpatient care. There is a need for urgent solutions, including communication guide, creation of common coping mechanisms and supporting measures [18]. 


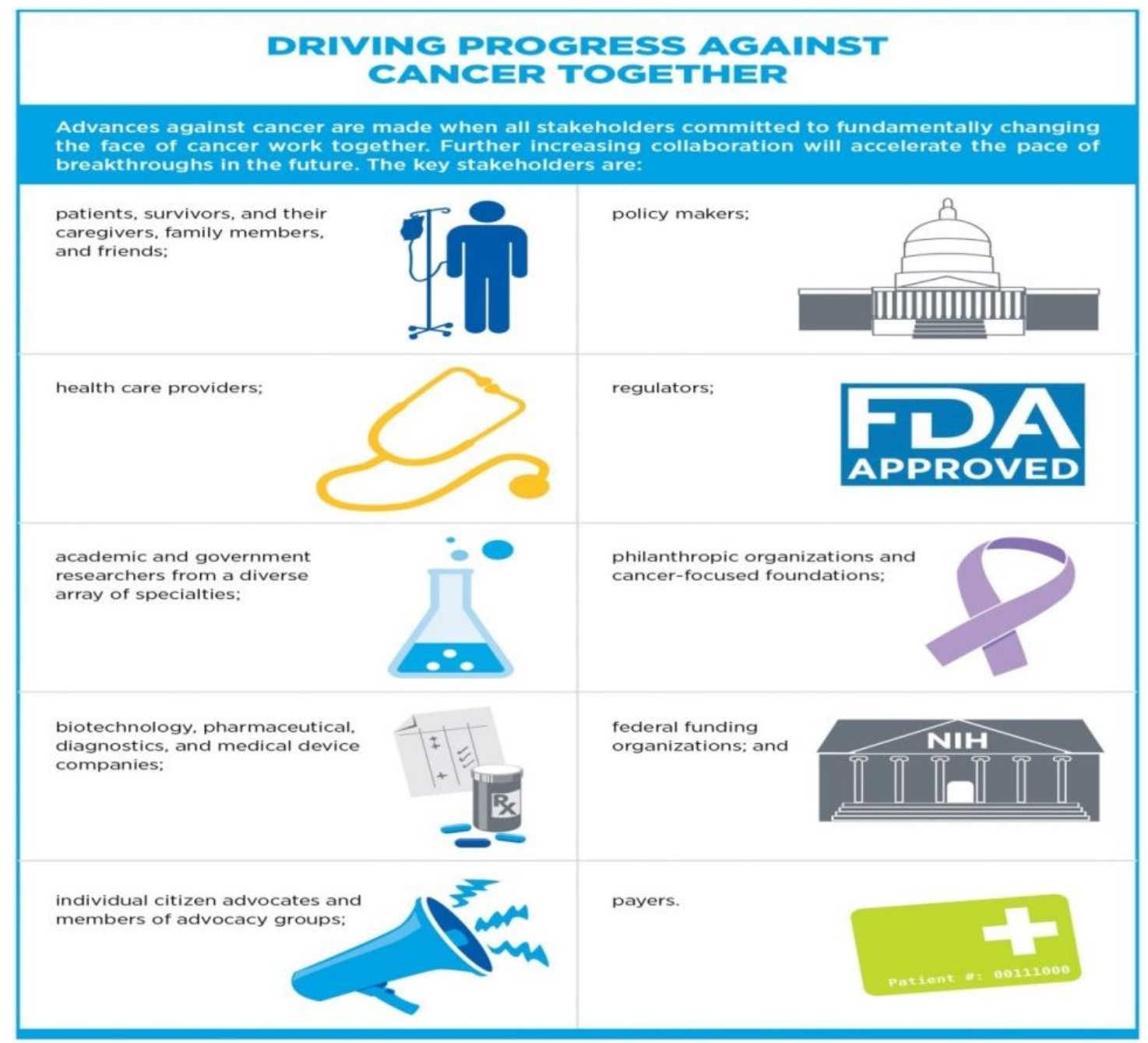

American Association for Cancer Research (AACR) Cancer Progress Report 2019

Figure 8. Stakeholders in the Management of Cancer Patients in Covid-19 Era

\section{Equitable Cancer Care in Pandemic Era}

It is important at this time to make noncommunicable disease prevention and control a global priority. Equitable cancer care in terms of priority regardless of income will improve cancer survivors. In achieving this, it is therefore important to work towards declaring universal healthcare to be a human right principle. $1 \%$ of all patients who have covid-19 had a history of Cancer. Cancer patients don't appear to have any benefit to testify of in terms of Health insurance in Nigeria, why is this so, and is anyone listening to them?

The global call by WHO in 2018 for urgent action towards elimination of cervical Cancer stimulated many low- and middle-income countries-(LMIC) in the pre-covid times to revise their national cancer control policies and commit resources to improve cancer screening cancel management [5].

The majority of the African countries had implemented their control policies before the incidence of the COVID-19. However, the COVID-19 induced health crisis is a potential threat to these LMIC initiatives.

The movement restrictions and lockdowns in various countries led to the slowing down of non-emergency services and the diversion of physical and manpower resources. This slowdown deeply impacted the entire continuum of cancer care [9].

Almost all African countries experienced suspensions in cancer screening which lasted at least one month owing to the lockdown restrictions, changing in health priorities, and 
reduced patient visits. This will no doubt widen the existing disparities in oncology care [10].

Since the re-opening of most cancer services in 2021, most of them generally operated at significantly reduced capacities [10]. There is an urgent need for high-level coordination efforts to ensure adequate continuity of cancer care following lockdown.

Restarting cancer screening activities as the crisis situation somewhat settles down will require a well-coordinated effort to reach out to the community more proactively [10].

Every COVID-19 patient with suspected Cancer should opt for getting an early cancer diagnosis and initiating treatment without delay. The benefits far outweigh the threats posed by COVID 19.

During the pandemic, hospitals may be unable to provide regular hospital service due to patient overload and inability to provide regular hospital services. In a report [11] from a questionnaire survey of 1147 patients with colorectal cancer, results showed that $78 \%$ of the patients were affected by the outbreak. The top three most affected events were laboratory and imaging examination, chemotherapy which included targeted therapy, and immunotherapy, the determination of treatment of shelves.

About half (63\%) of the patients visited the hospital for treatment, and among the patients who did not visit the hospital, $44.1 \%$ did not go because of travel restrictions or quarantine policies, to $3.4 \%$ because of fear of infection, and $10 \%$ because of cessation of outpatient services in the hospital [11].

Other research has shown that patients with Cancer are particularly vulnerable to respiratory viruses because of the immunosuppression caused by Cancer or anti-cancer treatments [12].

In another report [13], the significant heterogeneity among studies was seen for Cancer, $42 \%$ cases in the covid- 19 cohorts.

Cancer prevalence in the cohort from Asia was $3.9 \%$, Italy and USA were 5.1 and $5.2 \%$, respectively.
Cancer appears to be in risk factor for severe covid-19 infection. A higher prevalence of covid-19 infections rates has been reported in a number of studies with more severe outcomes in the cancer group $[14,15]$.

If the current pandemic takes longer or if the word encounters a re-emergence of the virus, our Cancer strategy will have to change, and cancer treatment pathways will have to adapt to the pandemic [13].

In New York City, a higher prevalence of cancer in patients with covid-19 infection was reported. $8 \%$ covid-19 patients admitted to the intensive care unit in Lombardy, Italy had a history of malignancy.

About $20 \%$ of covid-19 deaths across Italy were associated with active cancer patients [7, $16,17]$. In another article, only 10 patients $(0.9 \%)$ had cancer history and 3 patients had severe symptoms on admission amongst 261 reported patients in a cohort study [2].

Due to the description of non-essential services and reallocation of resources and at the same time the urgent global effort towards discovering therapies that treat or prevent covid19 infection, the shortening of traditional regulatory timelines has become the resultant effect.

This experience should stimulate similar health and government agencies in the way future cancer research is conducted, this, in turn, involves integrating patient perspective in our daily delivering care with an expectant patient satisfactory outcome.

Engaging patient experience in cancer care during the pandemic and beyond can help to separate signal from noise, consequently, the opportunities of having all of this information will create data and technology to provide meaningful and large-scale impact, which is of immense need today.

This gap is what this article seeks to bridge. Patients from across 4 major cancer treatment centres/facilities in the southwest were engaged with consent using a semi-structured interview. 
Their perspective on the experience and impact as cancer patients in the era of the COVID-19 pandemic in 2019/2021 regarding their cancer management during the peak of the pandemic was identified and presented in discourse form to provide informed decisions in the perception of the cancer patients as to the future of cancer management in the current era.

This way, the patient need not be afraid to seek care, i.e., negative for COVID-19 or positive for COVID-19. Patients should be confident that we are still here to care and listen to them.

\section{Objective}

There are three main objectives of this study.

1. To observe the level of cancer patient experience during the COVID-19 pandemic.

2. To observe the level of impact of the pandemic on their cancer management routine and,

3. To proffer possible measures at mitigating the observed impacts and improving patient experience during future pandemic occurrences.

\section{Methodology}

This study is a prospective case report which was implemented using a phenomenological qualitative approach where human experiences were reported through the descriptions that are provided by the people involved. A quantitative approach was also applied in terms of the statistics derived from the questionnaires used as well.

Patients from across 5 major cancer treatment centres / facilities in the southwest were engaged with consent using a semistructured interview and questioneers. The fivecancer treatment center includes Eko Hospitals PLC, Lakeshore Cancer Center, Olabisi Onobanjo University Teaching Hospitals (OOUTH) and Lagos University Teaching Hospital (LUTH), and Lagos State University Teaching Hospital (LASUTH).
Eko Hospitals PLC, Lakeshore Cancer Center, LUTH, and LASUTH are situated in Lagos State, OOUTH is at Ogun State. Eko Hospitals PLC and Lakeshore Cancer Center are private institutions. At the time of this study, all the centres used for this study were actively treating cancer patients and recognised as major cancer registry contributors in the southwest.

Their perspective on their experience as cancer patients about the observed impact of the COVID-19 pandemic on their cancer management between 2019/2021 was identified via semi-structured interview and use of questionnaires which is then presented in data form to provide informed decisions in the perception of the cancer patients as to the future of cancer treatment and patient satisfaction in the current era. This was mainly to collect appropriate information on some of the responses provided in an unpublished survey of 550 patients. The questionnaires were simultaneously filled in just as the interview was ongoing on the spot.

This allowed the participants to share their thoughts on the possible impact of the covid-19 outbreak through open-ended questions. Although it was challenging because of disclosure issues, however, interviews lasted between 15 minutes to an hour and was conducted in English and local languages. The results from the questionnaires were analysed using the Epinfo- $7^{\mathrm{TM}}$ version.

The summary of the responses was deliberated upon by presenting it to a sevenman panel of review. These responses and the resulting patient perspectives upon deliberation were further presented in a report thematic approach.

For strict ethical purposes, the name of the hospitals will be referred to as facilities A, B, C, D \& E, and in no particular order. Names of patients were withheld as the majority refused to consent to be recorded except for a few.

The guided structure questions are. 
1. How sudden was the occurrence of the COVID-19 pandemic to you? (Surprised/Normal/Both).

2. Did you expect that it would have any impact both on your status and treatment? (Yes/No).

3. Was the pandemic of any psychological effect to you? (Yes/No).

4. To what extent was the psychological effect during the lockdown? (Very severe/severe/normal).

5. In your opinion, what were the causes of the severe psychological effects?

6. Was there any care update by the clinic to you throughout the time of the lockdown? (Yes/No).

7. Was there any home visit, telephone call or online consultation through the period to you by your clinic representatives? (Yes/No).

8. What was your experience during your first visit to the clinic upon relaxation of the lockdown?

9. Did you notice strict adherence to covid-19 rules by health officials in the clinic (Yes / $\mathrm{No} /$ Partial).

10. What was your major challenge upon resumption of cancer treatment after the lockdown?

11. Did you have any health crisis during the lockdown and how did you manage it?

12. How satisfied are you with the measures your hospital took in terms of care during the lockdown and after the lockdown (Satisfactory/Non-Satisfactory).

\section{Results}

Of the 550 patients engaged in the unpublished thesis report on the policy integration of cancer patient experience, only 93 patients were accessed for this interview due to the limited time.

From the patients' response, it was obvious that the covid-19 pandemic occurred suddenly and with a shock to a majority of the cancer patients.
The impact of the pandemic actually became a reality to a majority of the patients after the initial months. They began to understand that their health was at risk, and access to treatment was obviously becoming a challenge.

Beyond just a mere shock, the impact of the pandemic became severely psychological, especially during the lockdown as testified by about $62 \%$. It was characterized with fear and constant worries following the search for an alternative to managing the resultant pains and crisis emanating from the unavailability of drugs. Actually, the major cause of the severe psychological effects was due to the resultant fear arising from the rumours of numerous deaths about the elderly sick ones and familiar cancer patients from other states and the inaccessibility to drug refill during the serial lockdowns.

We observed that, unlike the private cancer treatment centres, the public cancer treatment centres did little or nothing in terms of their cancer patient follow-up. In fact, most patients confirmed that reaching the cancer care providers was a challenge as a majority discovered that the customer/reception desk line wasn't even connecting at all. It became difficult accessing their care provider throughout the lockdown.

It was rather not surprising that only the Patients from the private treatment centres confirmed that drugs were sent to them using private commercial distribution services upon complaints made, A few had ambulances arrive their homes to pick them to the hospitals for closer observation when they had crises, but they confirmed it came at a cost as well.

Most patients reported that visiting the clinic for the first time after numerous weeks brought lots of nostalgic feelings. The atmosphere was a new normal, and its adherence to the new normal protocols wasn't optional. They observed the following activities, amongst other things at their clinics.

1.Compulsory Covid-19 protection strict measures. 
2.Not too-well coordinated activities in terms of clinic activities and appointments.

3.Huge percentage increase in cost expensive cost of treatment.

4.Staggered nature of health workers activities and,

5.Compulsory covid-19 test before commencement of further treatments.

Patients reported that there was very strict adherence to the covid-19 protection protocols initially; however, it appears a bit relaxed compared to the strictness observed months back.

They also reported to have observed other clinical issues as it affected their cancer treatment options, they include.

1.Long appointment date following various target clinic consultations.

2.An additional cost of laboratory test and compulsory covid-19 test before surgery and,

3.The increased cost of chemotherapy or radiotherapy treatment and drugs due to increased imputed hazard allowance of health officials and cost of PPE packages.

One notable observation during their clinic sessions was the confirmation of previous reports of the demise of many cancers' patients within the 4 months of the lockdown from various states. Regrettably, most of the deaths were reportedly associated with COVID-19.

According to a patient, she said "I am lucky to be alive because I suffered two crises in a row during the lockdown". Another 30\% of patients also confirmed that they had one or two resultant health crises during the lockdown and therefore are lucky to be alive as only a few could have access to the ambulance service in the private treatment centres.

Virtually all the patients came to realize that the effect of the covid-19 was worse than they thought, following the severe increase in the cost of treatment. The current hike in the cost of treatment has destabilised the hope of many patients. For instance, the cost of radiotherapy sessions is now approximately 246,000 Naira(512USD), and surgery is about a total of 550,000 Naira-(1,146 USD) (must be paid in two installments only), chemotherapy - drug administering cost about 50,000Naira. These prices represent a $40 \%$ increase from the previous cost. The covid-19 impact on the patients' cancer management routine as observed from the patient's perspective was not in doubt. It is far-reaching and at a huge cost.

The entire events and prevailing circumstances are clearly not unique to Nigeria alone; the observed level of cancer patient experience during the COVID-19 pandemic was illustrated in this study engagement. In fact, the little time scheduled for this study reduced the number of reached patients.

The results are summarily presented below (Table 2).

Table 2. Summary of Opened Structured Questions asked with Responses from Participants

\begin{tabular}{|l|l|l|}
\hline No & Questions and the summary response \\
\hline \multirow{2}{*}{1} & Question & How sudden was the occurrence off covid-19 to you? \\
\cline { 2 - 3 } & Summary Response & $\begin{array}{l}\text { Over 65 \% said the covid-19 came surprisingly, 15\% was actually expecting } \\
\text { the virus in Nigeria, therefore wasn't a surprise while 20\% said it was with } \\
\text { mixed feelings i.e., both a surprise and just normal feelings. }\end{array}$ \\
\hline 2 & Question & $\begin{array}{l}\text { Did you expect that it will have any impact both on your health status and } \\
\text { treatment? }\end{array}$ \\
\cline { 2 - 3 } & Summary Response & $\begin{array}{l}82 \% \text { didn't expect the impact as observed? Initially many felt no but after a } \\
\text { month of lockdown the reality became clear that certainly, their health } \\
\text { management was at risk. }\end{array}$ \\
\hline 3 & Question & Was the pandemic of any psychological effect to you? \\
\hline
\end{tabular}




\begin{tabular}{|c|c|c|}
\hline & Summary Response & $\begin{array}{l}\text { Over } 62 \% \text { said yes' except for those who had recently seen their doctor and } \\
\text { had their drugs. However, when their drugs finished within two months with } \\
\text { no access to drug due to restricted movement, the psychological effect began } \\
\text { to emerge with obvious panic. }\end{array}$ \\
\hline \multirow[t]{2}{*}{4.} & Question & To what extent was the psychological effect during the lockdown? \\
\hline & Summary Response & $62 \%$ said it was actually severe for various reasons. \\
\hline \multirow[t]{6}{*}{5.} & Question & In your opinion, what were the causes of the severe psychological effects? \\
\hline & \multirow[t]{5}{*}{ Summary Response } & $\begin{array}{l}\text { The resultant fear arising from the rumours of numerous deaths about the } \\
\text { elderly sick ones and familiar cancer patients from other states. }\end{array}$ \\
\hline & & $\begin{array}{l}\text { Fear of the lack of accessibility to treatment (chemotherapy and target } \\
\text { radiotherapy sessions). }\end{array}$ \\
\hline & & $\begin{array}{l}\text { Inaccessibility to drug refill when exhausted thereby having the experience } \\
\text { of unabated excruciating pain. }\end{array}$ \\
\hline & & Cancellations of appointments. \\
\hline & & Fear of exposure to the covid-19 virus. \\
\hline \multirow[t]{2}{*}{6.} & Question & $\begin{array}{l}\text { Was there any follow up- care update by the clinic to you throughout the } \\
\text { time of the lockdown? }\end{array}$ \\
\hline & Summary Response & $\begin{array}{l}14 \% \text { said "Yes" and } 86 \% \text { said "No". Almost all the patients that explained } \\
\text { that nothing as to a single follow-up or check up on them was experienced } \\
\text { were from the public treatment centres unlike the patients from the private } \\
\text { treatment cancer centres that had more of positive responses, However, the } \\
\text { majority complained that the intervention came quite late in the heat of the } \\
\text { pandemic. }\end{array}$ \\
\hline \multirow[t]{2}{*}{7.} & Question & $\begin{array}{l}\text { Was there any home visit, telephone call or online consultation through the } \\
\text { period to you? }\end{array}$ \\
\hline & Summary Response & $\begin{array}{l}\text { Only the Patients from the private treatment centres confirmed that drugs } \\
\text { were sent to them using private commercial distribution services upon } \\
\text { complaints made, A few had ambulances arrive their homes to pick them to } \\
\text { the hospitals for closer observation when they had crises, but they confirmed } \\
\text { it came at a cost as well. }\end{array}$ \\
\hline \multirow[t]{7}{*}{8.} & Question & $\begin{array}{l}\text { What was your experience during your first visit to the clinic upon relaxation } \\
\text { of the lockdown? }\end{array}$ \\
\hline & \multirow[t]{6}{*}{ Summary Response } & they observed the following activities amongst other things. \\
\hline & & Compulsory Covid-19 protection strict measures. \\
\hline & & $\begin{array}{l}\text { Not too-well coordinated activities in terms of clinic activities and } \\
\text { appointments. }\end{array}$ \\
\hline & & Huge percentage increase in cost expensive cost of treatment. \\
\hline & & Staggered nature of health workers activities. \\
\hline & & Compulsory covid-19 test before commencement of further treatments. \\
\hline \multirow[t]{2}{*}{9.} & Question & $\begin{array}{l}\text { Did you notice strict adherence to covid-19 rolls by health officials in the } \\
\text { clinic (Yes / No /Partial). }\end{array}$ \\
\hline & Summary Response & $\begin{array}{l}\text { Majority said "Yes". There was very strict adherence initially; however, it } \\
\text { appears a bit relaxed compared to the strictness observed months back. }\end{array}$ \\
\hline 10. & Question & $\begin{array}{l}\text { What was your major challenge upon resumption of cancer treatment after } \\
\text { the lockdown }\end{array}$ \\
\hline
\end{tabular}




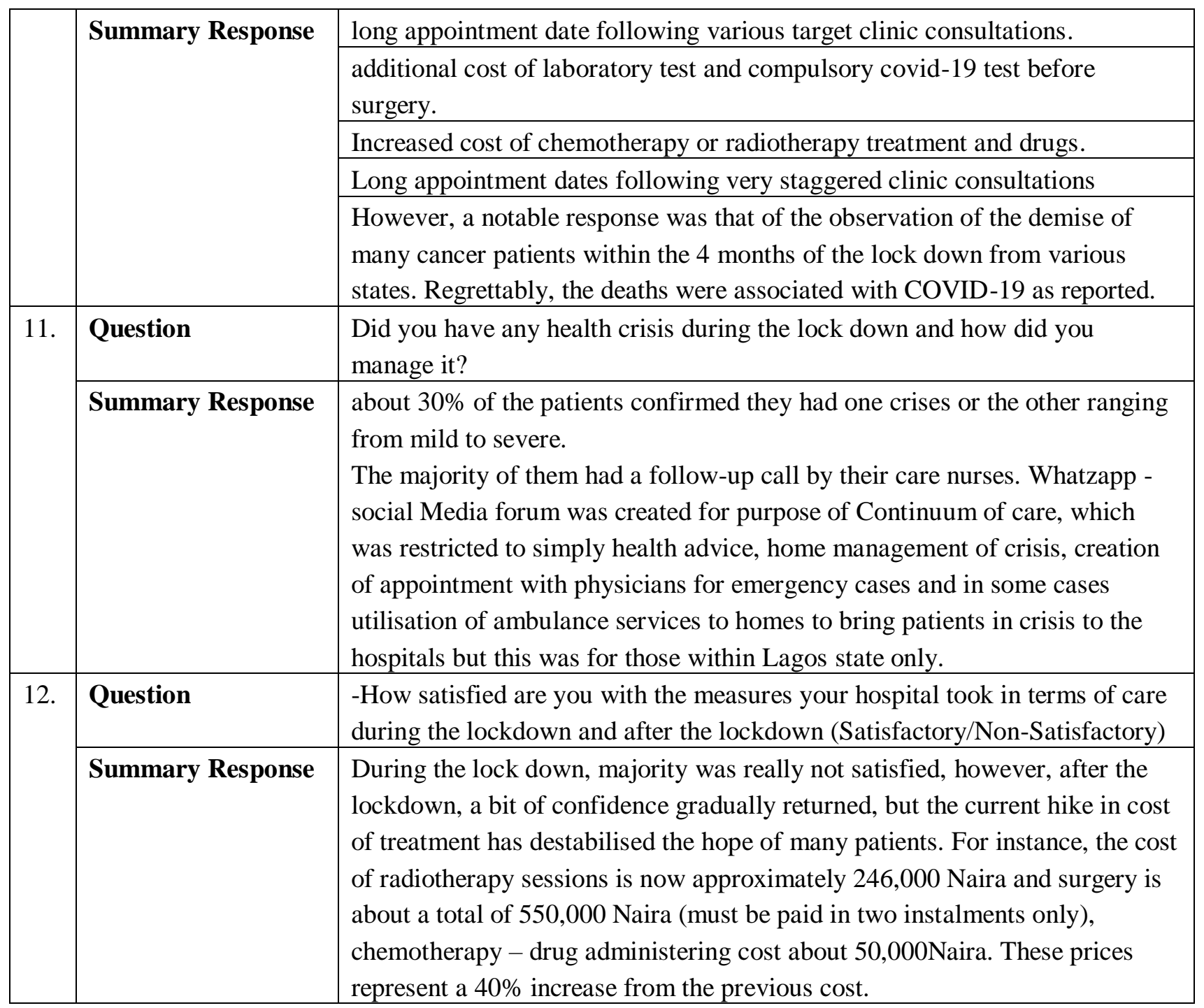

\section{Discussion}

A majority of the cancer patients are concerned about the events of the outbreak and the lasting effect on the re-start and normalisation of both cancer screening and treatment services.

As at the time of this research, covid-19 patients with cancer symptoms would most probably experience delay or difficulty accessing diagnostic and treatment services, especially in lower and middle-income countries, and this will most possibly aggravate the situation with greater impact on the socioeconomically disadvantaged population [10].

Most papers reported that the screening test in diagnostic and treatment services in most African countries where worse affected [5], and this report is in agreement with this studies' findings.

There is a need for urgent new cancer management strategies to be adopted in order to maintain unhindered services during the acute phase of the pandemic and its aftermath.

A newly adopted strategy engaged from the grass-root level can significantly enhance the quality of treatment as well as the reach of cancer screening. Of most concern is the need to decentralize health screening services to primary care centres in rural primary health areas because the presence of this screening centres are major hub for awareness campaigns within the geographical area.

A system galvanized towards telemedicine via ICT technology will be very helpful in easing access to patients to aid monitoring. Regardless, if the cancer centre is a public or 
private owned centre. Restarting and maintaining cancer management activities as the pandemic relapses will deliberately require a well-organized effort to galvanize a strong community reach. The concerns of the apparently healthy individuals are similarly concerns to public health professionals worldwide. The need to aid their return to routine health care services is more important and therefore requires an urgent reorganization of cancer management services.

There is an urgent need for adequate structural analysis to enumerate the impact of the pandemic from; health systems perspectives, focusing on governance, finance, workforce, infrastructure and services, information system, and quality assurance process relevant to screening Continuum.

A focus in the increased awareness of cancer screening and proposed cancer management policy intervention is strategically crucial, especially during the pandemic, as this will build public trust. The current fears of perception of the common public of their personal risk of severe illness from covid-19 versus the risk of not seeking healthcare advice if they have symptoms suggestive of Cancer needs to be changed [10, 18, 19].

Suffix this study to have shown that the covid-19 impact on the patients' cancer management routine as observed from the patient's perspective was not in doubt. It is farreaching and has come at a huge cost. The entire events and prevailing circumstances are clearly not unique to Nigeria alone; the observed level of cancer patient experience during the COVID-19 pandemic was illustrated in this study to a reasonable extent much resourceful and dependable. [2, 7, 11, 14-18].

In the response review panel of six core health workers and one Female cancer survivor, the various responses were discussed, and possible interventions were proposed for future strategic cancer management in the pandemic era bearing in mind that it is no longer business as usual. Amongst the responses, the review team was strongly burdened with unpleasant and unfortunate deaths reported following the lockdown effect, especially from patients who largely reside in rural municipals of other states outside Lagos across Nigeria but routinely visited cancer treatment Facilities in Lagos.

Furthermore, the much unprepared state-ofthe-nations health care system towards existing diseases in terms of neglect and diversion of attention and resources to combat covid-19 was discussed and solutions proffered. The upsurge in the cost of treatment was also another concern. There is an urgent need to have new strategies and national policies to guide practices during pandemics by Nigeria. Amongst many decisions conversed. A few noteworthy among these include:

\section{Re-orientation /Pandemic Control}

Emphasis should be placed on adequate treatment and re-orientation of cancer patients regarding the pandemic occurrence using past cases as a reference to be a coping strategy. These can be disseminated via social media platforms which are easily accessed by patients using smart phones etc.

\section{Staff Training and Retraining}

Private and Public Hospitals/clinic management must resolve to train and retrain staffs of cancer screening centres to prevent discontinuation of cancer screening and treatment services during pandemics.

\section{Awareness Campaign}

Awareness at all levels is a priority. Therefore, a deliberate policy to drive monitored Improvement on community outreaches using possible mobile clinics on dedicated ambulances and to expand screening facilities to rural areas should be established without further delay.

\section{Screening and Result Accessibility}

The clinic's information technology departments should Innovate means of delivering screening test results privately online 
and follow-up consultation online during pandemics.

\section{Special Pandemic Services}

All Public health Nurses and workers should be engaged and trained to be able to deliver home visits and delivery of drugs during pandemic periods.

\section{Adequate Mobilisation of Manpower}

Despite reassigning dedicated cancer screening and treatment staff to covid-19 related duties, proper delegation of duties as to proper care for cancer patients must be ensured.

\section{Adequate Amenities}

Personal protective equipment (PPE) must be provided for public health workers involved in visitation and public assignments.

\section{Adequate Routine Supply}

Routine provision of adequate hand sanitizers and masks for the health workers and screening participants, and patients should be ensured.

\section{Proper Stock Management}

Proper adequate stocking of PPEs, drugs, and hand sanitizers should be planned, and resources made available on time.

\section{Committed Governance}

A strong effective governance must be committed to maintain implementation directives and cancer control services, especially during the pandemic.

\section{Improved Turnaround Time}

Despite an active leadership capacity, timely and efficient planning to ensure the continuity of routing services (diagnostic and treatment) is paramount.

\section{Monitoring and Evaluation}

It is mandatory for Public and Private Cancer clinics to establish a monitoring and evaluation (M\&E) committee at this juncture. They are expected to be saddled with the ad-hoc responsibility of implementing the addressed themes and integrating the Patient perspective into local policy using the Patient perspective integration Framework.

\section{Summary}

This study showed that the covid-19 impact on the patients' cancer management routine as observed from the patient's perspective in this study was not in doubt. It is far-reaching, and it came at a huge cost. It also illustrated that the entire events and prevailing circumstances are clearly not unique to Nigeria alone; the observed level of cancer patient experience during the COVID-19 was highly informative and dependable as it collaborated with other reports from other centres.

It is possible for Health workers, upon engaging patient perspective, to show a good level of performance knowledge.

The Patients have no doubt come to terms and awareness on the benefit of handwashing, use of nose masks, and social distancing against viral transmission as it has become a routine following the strict covid-19 measures.

More patients have also developed a wellinformed sense of validation concerning maintaining hygiene standards.

The pandemic, somehow in disguise, presented the valuable perspective of Life reflection and gratitude as reflected by patients in their resolve to appreciate God for each day experienced.

The language and attitude of a majority of patients engaged was largely that of a deep reflection of the privilege of having access to early diagnosis, treatment, and adequate support; however, the complaints of the past, knowing that they are alive and have hope to keep fighting on with gratitude and sense of acceptance.

A few patients expressed their desire to clearly opt-out of traditional treatment applied during the lockdown and to resume orthodox treatment. However, they are worried of the 
possibility of nosocomial infection and the huge cost implication.

Others also wished that the experience of the social media engagement platform should continue as it provided a good level of the bridge of the gap of information.

The cost of treatment remains a major concern as it largely translates to possible dropout from treatment courses for most cancer patients who can't afford the current cost of $\$ 470$ per session of radiotherapy and $\$ 1,150$ (at two installment payments) for surgery.

\section{Conclusion}

The COVID-19 pandemic was sudden, and the impact on both economy and the current global health institution has been devastating. The disease turned out to be evidently associated with cancer disease and therefore has assumed a huge threat to the cancer patients as well as their management. Cancer Patients had a terrific time pulling thru the nationwide lockdowns till the subsequent relaxation. The concerns of the cancer patients and survivors during and after the covid-19 pandemic are similarly a concern to public health professionals worldwide. The need to aid their return to routine health care services is more important and therefore requires an urgent reorganization of cancer management services.

Finally, this study reflected a very huge level of poor cancer patient experience in the public cancer treatment centres while the reverse is the case with the private cancer treatment centres. This can be managed if an urgent intervention as proffered is implemented.
The urgent intervention should be focused on patient re-orientation/pandemic control, staff train and retraining, awareness campaign, screening and result accessibility, special pandemic services, adequate mobilisation of manpower, adequate amenities, adequate routine supply, proper stock management, improved turnaround time, monitoring \& evaluation and committed governance.

\section{Conflict of Interest}

There is no conflict of interest.

\section{Acknowledgements}

In keeping with the ethical practice in reporting scientific information, due acknowledgements have been made wherever the findings of others have been cited.

I humbly acknowledge the role and support of all members of the Olabisis Onobanjo University Ethics Research Committee.

The Laboratory Scientist of the Department of Laboratory Services OOUTH, especially in the Chemical Pathology/Immunology Unit.

Members and Staff of the Oncology Department at Eko Hospitals PLC.

Members and Staff of the Oncology Department at Lake Shore Cancer Centre.

Members and Staff of the Oncology Department at Lagos University Teaching Hospitals.

Members and Staff of the Oncology Department at Olabisis Onobanjo University Teaching Hospitals.

Members and Staff of the Oncology Department at Lagos State University Teaching Hospitals. 


\section{References}

[1] World health organization 2020. WHO coronavirus disease (covid-19) dashboard. https://covid-19.who.int/ Accessed Aug. 21, 2021.

[2] Dong Shuang, Luo Chenggang, Hu Xuebo, Zhang Jing, Cai Qian, Qian Yu, Ran Fengming, Ou Wuling, Wang Jun, Huang Qing, Ren Tianhua, Han Guang, Zhang Feng, Wei Wei, Liang Xinjun, Xu Huiting, Wang Sheng, Shi Lulu, Wei Shaozhong, Hu Sheng. (2020). Expert Consensus for Treating Cancer Patients During the Pandemic of SARSCoV-2. Frontiers in Oncology. 10, 2020;1555. https://www.frontiersin.org/article/10.3389/.2020.01 555 Doi: 10.3389/fonc.2020.01555.

[3] Coronado, A.C.; Tran, K.; Chadder, J.; Niu, J.; Fung, S.; Louzado, C.; Rahal, R. in collaboration with the System Performance Steering Committee and the Technical Working Group (2017). The Experience of Patients with Cancer during Diagnosis and Treatment Planning: A Descriptive Study of Canadian Survey Results. Curr. Oncol. 2017, 24, 332-337. https://doi.org/10.3747/co.24.

[4] Bailey C, Black JRM, Swanton C. (2020). Cancer Research: The Lessons to Learn from COVID-19. Cancer Discov. 2020 Sep;10(9):12631266. doi: 10.1158/2159-8290.CD-20-0823. Epub 2020 Jul 15. PMID: 32669285.

[5] Franz Geisslinger, Angelika M. Vollmar and Karin Bartel. (2020). Pharmaceuticals 2020, 13, 143; doi:10.3390/ph13070143.

www.mdpi.com/journal/pharmaceuticals Accessed june. 21, 2021.

[6] Kaufman HW, Chen Z, Niles J, Fesko Y (2020). Changes in the Number of US Patients with Newly Identified Cancer Before and During the Coronavirus Disease 2019 (COVID-19) Pandemic. JAMA Netw Open. 2020;3(8): e2017267.

[7] Al-Shamsi H O, Alhazzani W, Alhurayi A (2020). A practical approach to the management of cancer patients during the novel coronavirus disease 2019 (COVID-19) Pandemic: An International collaborative group. Oncologist 2020;25(6); e936e945).

[8] Langarizadeh M, Tabatabaei MS, Tavakol K, (2017). Telemental Health Care, an Effective
Alternative to Conventional Mental Care: A Systematic Review. Acta Inform Med 2017; 25:240. [9] Bouanani N, Bendari M, Naim A, (2020). Managing cancer center during the SARS-COVID 19 Pandemic. Morocan experience. Asian Pac J cancer care 2020; 5(S1):219-223.

[10] Villian P, Carvalho A. L, Lucas E, (2021) Cross-sectional survey of the impact of the covid-19 pandemic on cancer screening programs in selected low- and middle-income countries; study from the IARC COVID-19. Impact study group. Int.J cancer 2021: 149, 97-107. https://doi.org/10.1002/ijc33500. [11]Zhaoya Gao, Yong Yang, Changmin Dmg, pengfei niu, wenshang huang, fuming lei, jin gu, (2020). Oncologist perspective: when Cancer encounters COVID-19. The oncologist 2020:25:25:61423.

[12] Kamboj M., Sepkowitz KA, (2009). Nocosomial infections in patients with cancer Lancet Oncol. 2009.

[13] Kristof Nemeth, Loannis Nikolopoulos, Ali Reza Mani, (2020). Scooping review on the prevalence of Cancer in COVID-19 Patients. BJS 2020; 107: e456-e457.

[14]Wang D, Hu B, Hu C, Zhu F, Liu X, Zhang, (2020). Clinical characteristics of 138 hospitalized patients with 2019 novel coronavirus infected Pneumonia in Wuhan China JAMA 2020; 323: 1061 -1069 .

[15]Epidemiology Group of emergency response mechanism of new coronavirus Pneumonia; Analysis of epidemiological characteristics of new coronavirus Pneumonia. https://doi.org/10.3760/cma.j.issn.02546450.2020.02.003.

[16] Sharma A. Malviya R, Kumar V, Gupta R, Awasthi R. (2020). Severity and Risk of Covid-19 in cancer patients: An Evidence-based learning, dermatologic therapy 2020;33; e13778; https://doi.org/10.111/doh.13778.

[17] Uzzo RG, Kutikov A, Geymsman DM, (2019). Coronavirus disease 2019 (COVID-19); Cancer during the pandemic. http://www.uptodate.com/contents/coronavirusdisease-2019-covid-19-cancercare-during-thepandemic Accessed on May 23, 2021. 
[18] World health organization, primary health care on the road to universal health coverage: 2019, global monitoring, Report, Geneva WHO: 2019. Accessed June. 21, 2021.

[19]Beddoe AM. (2019). Elimination of cervical cancer: challenges for developing countries. E. cancer medical science. 2019:13:975.
[20]Henry A. O and Mgbeafuluba I. C. (2018). Awareness and Practice of Cervical Cancer Screening and Human Papilloma Virus Vaccination (HPV) Uptake among Female Staff in Private Tertiary Hospital in Lagos Nigeria Texila International Journal of Public Health Volume 6, Issue 1, Feb 2018. 